\title{
Two Thermocouples Low Power Wireless Sensors Network
}

PhD Vytautas MARKEVICIUS ${ }^{\mathrm{a}}$, PhD Dangirutis NAVIKAS ${ }^{\mathrm{a}}$, PhD Darius

ANDRIUKAITIS ${ }^{a *}$, MSc Mindaugas CEPENAS ${ }^{a}$, PhD Algimantas VALINEVICIUS ${ }^{a}$, PhD Mindaugas ZILYS ${ }^{a}$, PhD Reza MALEKIAN ${ }^{\mathrm{b}}$, PhD Arturas JANELIAUSKAS ${ }^{\mathrm{a}}$, PhD Wojciech WALENDZIUK ${ }^{c}$, PhD Adam IDZKOWSKIc

aDepartment of Electronics Engineering, Kaunas University of Technology,

Studentu St. 50-438, LT-51368 Kaunas, Lithuania

bepartment of Electrical, Electronic and Computer Engineering, University of Pretoria, Pretoria, South Africa

'Department of Electrical Engineering, Bialystok University of Technology, Wiejska 45D St., 15-351 Bialystok, Poland

E-mails: vytautas.markevicius@ktu.lt; dangirutis.navikas@ktu.lt;

darius.andriukaitis@ktu.lt*(correspondingauthor); mindaugas.cepenas@ktu.edu; algimantas.valinevicius@ktu.lt; mindaugas.zilys@ktu.lt; reza.malekian@ieee.org; arturas.janeliauskas@ktu.lt; w.walendziuk@pb.edu.pl, a.idzkowski@pb.edu.pl 
Abstract: This paper presents technologies and experiments of a wireless sensors using two thermocouples network. It was established that the energy consumption during sensor measurements is usually up to 10 times lower compared to the energy consumption at the time of establishing wireless connection for most protocols. For this reason, new simplified wireless connection protocol was created.

Extremely low energy wireless sensor hardware and software equipment was designed. The newly created universal measurement module allows the use not only thermocouples, but also various types of analogue sensors, thermocouples, pressure bridges, Resistance Temperature Detectors (RTD) and digital sensors communicating through SPI or I2C interface. The newly designed specific power supply scheme allows to supply the sensor and radio module with the voltage from $1.2 \mathrm{~V}$ to $3.6 \mathrm{~V}$ batteries. When conducting periodic measurements every second, the use of newly designed hardware and software equipment enables the wireless sensor to be operated for up to 3 years from two 1200 mAh capacity batteries.

Keywords: thermocouples, temperature, measurement, sensor, wireless, WSN, network

\section{Introduction}

Temperature is certainly among the most commonly measured parameters in industry, science, and academia. Recently, the growth of wireless instrumentation technology, along with some clever innovations, has provided new ways to apply temperature measurement sensors combined with personal computers to collect, tabulate, and analyze the data obtained. For complex, multi-sensor applications, wireless devices provide a means to eliminate the nuisance of running multiple leads over long distances through harnesses or conduit to a control room, instrument panel, or equipment rack, while keeping track of which leads are which. For simpler one or two sensor applications, it means installing the wireless sensor, setting up the receiver, and being done [1]. 
The flexibility and connectivity of wireless networks are the factors which have significantly contributed to the increase of their popularity. Wireless sensor networks have become highly relevant in processes such as system monitoring, actuation, target tracking, automation and process control [2]-[8]. Wireless sensor networks have proved to be irreplaceable when overcoming the costs and technical difficulties associated with the use of wired networks [9], [10]. When it comes to the wireless communications, one may choose from a great variety of protocols [2]-[4], depending on the specific needs associated with the application. Regardless of the specific geographical or geopolitical factors observed in various countries around the globe, one of the essential issues associated with the use of such networks remains environmental monitoring and conservation. The goal of this work is to create a wireless network consisting of various types of sensors (temperature, humidity, pressure, etc.) whilst ensuring minimal wireless sensor energy consumption. This would allow to operate such network with mobile energy sources such as batteries or accumulators for at least several years.

\section{Related Works}

Different manufacturers now offer a great variety of radio modules which support different data exchange protocols, however, as concerns the realization of wireless sensors, the most commonly encountered protocols and supporting equipment for short-range radio connectivity are ZigBee, Bluetooth and ANT. There are also different Bluetooth generations and their versions for specific solutions to consider. Taking into consideration the need to ensure low energy consumption, Bluetooth v4.0 Low Energy [11], [12] (Bluetooth SMART) version was chosen as the most relevant for designing the new wireless sensor system. There are also known low-power, wide-area (LPWA) technologies, e.g. LoRa, SigFox [18].

When transmitting a small amount of data (up to 10 bytes) using standard protocol (Bluetooth, ZigBee or ANT) the duration needed to establish a data exchange session can be several (and even ten or more) times longer than that needed to transmit the actual data. The 
energy consumption during the process also increases accordingly. Bluetooth Low Energy pairing takes long time, after that further process is going energetically efficient, and transmission takes a short period. The main drawback of the Bluetooth Low Energy is a short range [13] and it transmits a lot of redundant data, which are necessary in order to maintain the universality. In many cases the Bluetooth, ZigBee and ANT technologies (protocols) used for transmitting data are neither necessary nor simple, and an energetically efficient radio module combined with simple communication protocol would be enough. Based on the research [14, 18], comparison of peak power requirements for various technologies was provided, and for BLE is $12-15 \mathrm{~mA}$, ANT - $17 \mathrm{~mA}$, ZigBee varies from 17.6 to $29 \mathrm{~mA}$, LoRa $-32 \mathrm{~mA}$. Another research [15] regarding connection time was analysed. The following conclusion were made: ANT, Bluetooth SMART and ZigBee connection technologies are not optimal for this particular type of device due to the prolonged time necessary to establish connection. The empirical research results indicate that in the case of Bluetooth Low Energy, the time it takes to establish connection is $1150 \mathrm{~ms}$, in the case of ZigBee - $250 \mathrm{~ms}$ and in the case of ANT $930 \mathrm{~ms}$. Meanwhile, the time that is needed to measure temperature, humidity or pressure is only from 1 to $10 \mathrm{~ms}$ and the time that is needed to send out the data is from 1 to $5 \mathrm{~ms}$. Therefore, a decision was made to design own data exchange protocol. This protocol used only seven bytes (packet length, device address, packet ID, CRC) to ensure addressing and security and therefore achieved very efficient data transmission. The receiver acknowledges each transmitted packet to increase reliability of communication. The number of bytes is lower when comparing our protocol with SigFox, which is considered as lightweight protocol for small messages. SigFox frame contains 14 bytes of protocol overhead and up to 12 bytes of data payload. However, apart from two temperature values the SigFox messages can contain other data e.g. GPS coordinates. 
The radio module chosen for wireless data transmission was the A110LR09 (produced by Anaren) which can be operated in the frequency range of $868-870 \mathrm{MHz}$ or $902-928 \mathrm{MHz}$. This radio module is based on CC110L chip which has good ratio of power consumption over achievable range (distance up to $500 \mathrm{~m}$ with non-directional antenna), fast start-up time (up to $240 \mu$ s from sleep to TX or RX mode), possibility to implement custom protocol, and belongs to a budget line of sub-1 GHz RF Transceivers.

\section{Experiments}

\section{A. Wireless sensor}

The newly designed system comprises wireless sensors capable of reading, processing and transmitting measurement data from sensors via wireless connection. The data sent via wireless sensors is directly received by the concentrator where that data is structured and prepared for further transmission prior to interpretation.

Wireless sensors are supplied with energy from limited capacity energy sources; therefore, the design must be in accordance with the objective to reduce energy consumption as much as possible. This can be achieved by using low energy components, introducing a specific wireless data exchange protocol and ensuring that the majority of time the wireless sensor is kept in the sleep mode. The system uses the star topology of network and each wireless sensor sends data directly to the concentrator. It can communicate with up to 255 wireless sensors. It foresees the use of a single connection channel only, so the data exchange can take place only in a single direction at a time. Wireless sensors are programmed to be activated a certain intervals of time to carry out measurements and send out the acquired data. The wake up times among distinct sensors are not synchronized, therefore, there is a small but realistic chance of conflict when several wireless sensors are sending out data at the same time. In such cases the concentrator may fail to receive the data sent or reject such data due to interference. In order to protect from 
such event, it acknowledges received data by sending response to wireless sensor. Individual sensors are recognized and connections are established using identification code system.

Texas Instruments MSP430G2553 microcontroller was selected for the wireless sensor. This microcontroller stands out for its minimized energy consumption, fast wake up times and flexible clocking architecture. Despite its low cost it offers two communication ports. One of them is used for communication with the wireless sensor via the SPI interface whilst the other is reserved for communication with the digital sensors.

Most sensors require voltage which is higher than the lower limit of wireless sensor. For this reason, the system foresees a supply scheme which can boost the supply voltage. To serve this particular purpose TPS61221 integrated switch was used. It allows to reach a relatively high energy conversion efficiency at low voltage loads [16]. With negligible outlet load this device consumes a current of $5 \mu \mathrm{A}$ only. Moreover, it requires very few external components. The option of fixed $3.3 \mathrm{~V}$ output voltage was selected to eliminate the need for external resistance divider which is otherwise necessary. However, upon using a voltage multiplier all the time, it significantly increases energy consumption. This has led to the decision to realize two possible supply modes which could be controlled using a microcontroller: direct - straight from the energy supplying element and indirect - by using a voltage booster. In the case, the microcontroller detects that the battery voltage is insufficient, it can switch the supply chain from the direct to the indirect mode one and vice versa. This allows to significantly extend wireless sensor operation time from limited power source.

The newly designed wireless sensor prototype is modular and can be combined with various extensions as seen in Figure 1. For temperature measurement ADS1118 converter extension module for $\mathrm{K}$ type thermocouple is used. 


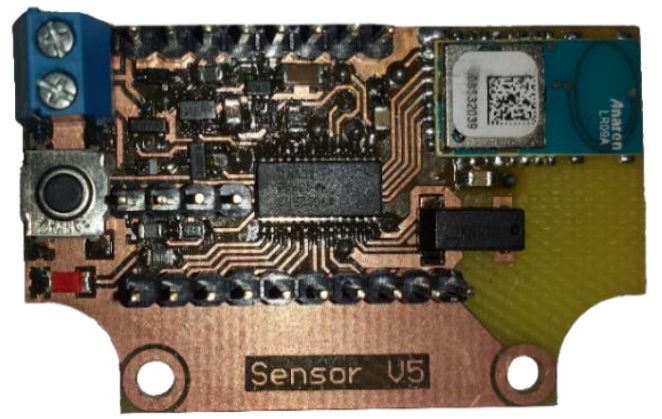

(a)

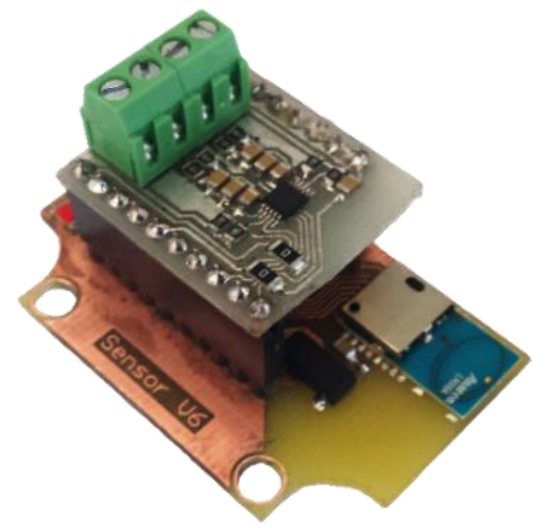

(b)

Figure 1. Wireless sensor prototype: (a) main sensor module, (b) ADS1118 thermocouple extension module mounted on wireless sensor.

The largest portion of firmware operations are executed in the main cycle. The algorithm block scheme is shown in Figure 2. 


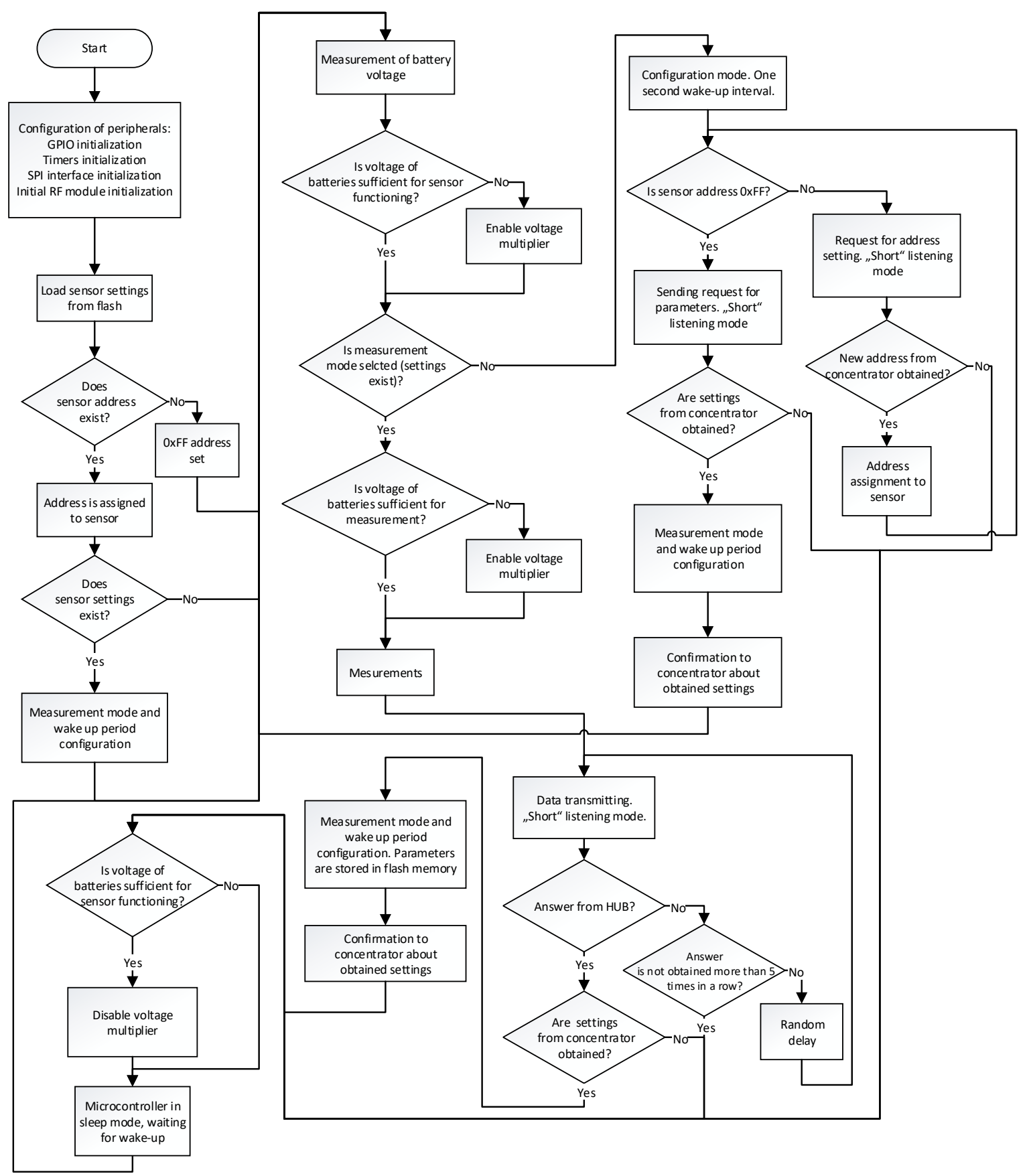

Figure 2. Wireless sensor operation block algorithm.

Due to the necessity to maintain the lowest energy consumption possible, the pauses which take a longer time are realized by putting the processor to the sleep mode and returning it to the ordinary mode using timers. For most of the time the microprocessor is kept in the Low power mode 3 . In the meantime, timers are supplied with the clock frequency by the externally 
attached crystal oscillator $(32768 \mathrm{kHz})$. A microcontroller operating in such a mode uses approximately $0.7 \mu \mathrm{A}[17]$.

\section{B. Concentrator}

With the aim to extend the capabilities of concentrator use, the communication of the microcontroller via the interfaces RS-232, RS-485 and USB via UART was foreseen. The concentrator can be combined with various industrial equipment used for reading and processing all types of data. The main components, comprising the concentrator are displayed in Figure 3.

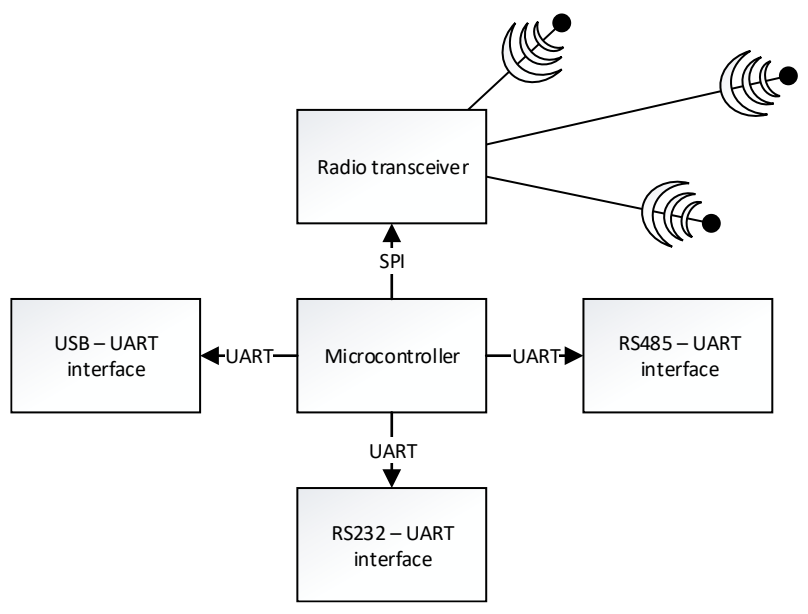

Figure 3. Components comprising the concentrator.

The microcontroller architecture chosen for the concentrator was ARM Cortex M4 (STM32F303CCT6 produced by STMicroelectronics). This decision was based on the high efficiency of microcontrollers of this architecture, which is very important in terms of speed when it comes to processing the data received. Because of the decision to store all data received from wireless sensors in the microcontroller, another major criterion was the amount of SRAM memory. Another features of interest were Real-time clock RTC support for accurate timestamps of data reception and hardware Modbus RTU protocol communication support. 
The prototype of the concentrator is shown in Figure 4.

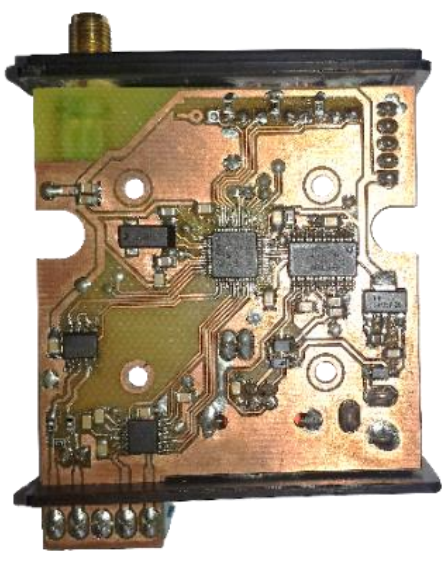

(a)

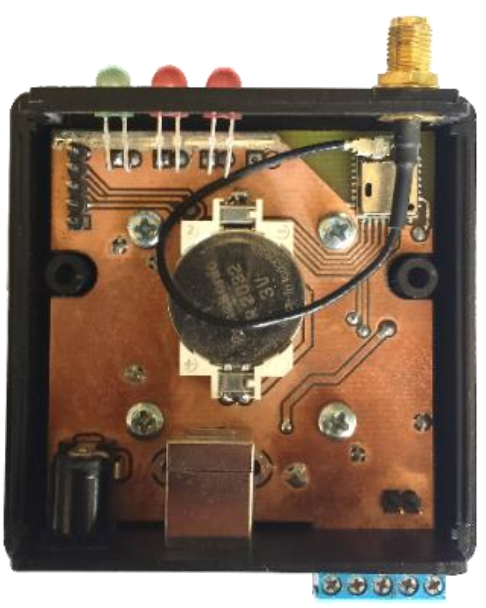

(b) 


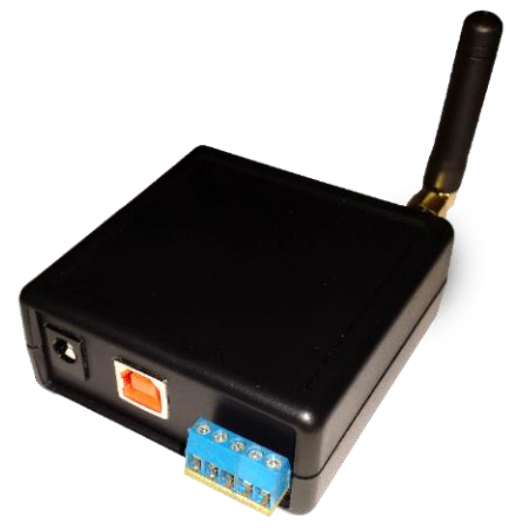

(c)

Figure 4. The main components comprising the concentrator: (a) top view of PCB,

(b) bottom view of PCB, (c) general view.

The newly designed concentrator software consists of two parts - the main cycle and the tasks being completed in the interrupt initiated upon receiving a message via the radio network. The block scheme of the main cycle is provided in Figure 5. After receiving an interrupt from radio module, the algorithm shown in Figure 6 is executed. Upon launching the program, the first operations which take place are the configuration of peripheral equipment and the making of all necessary data structures. Afterwards the intervals are enabled and the mode of radio network is activated. The concentrator spends most of the time in this particular mode, i.e., it is constantly awaiting new data to be sent from the wireless sensors. It switches to the sending mode only for a short period of time after receiving a portion of data in order to transmit acknowledgment about received data. 


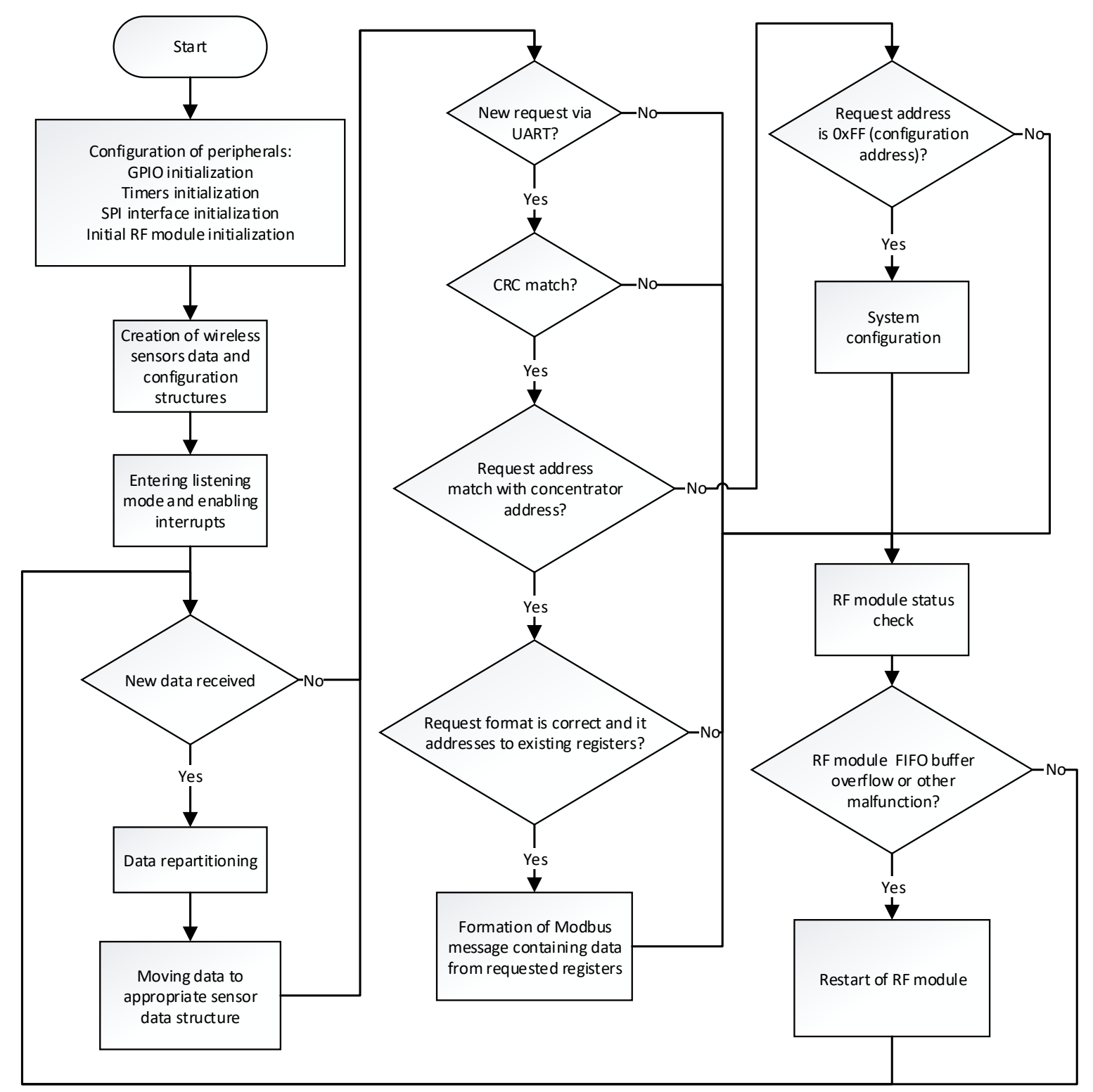

Figure 5. The block scheme of the main cycle of the concentrator software.

Every wireless sensor has a byte unique identification address (from 0 to 255) which enables the concentrator to determine whether it is being served and where the portion of the SRAM memory dedicated to that particular sensor is located. The memory for all wireless sensors is assigned in the form of structure array, where every element in the mass is a structure allocated to a separate wireless sensor. This mass is comprised of 255 structures and the number of each structure corresponds with the address of the allocated wireless sensor. The data 
received from a wireless sensor is first processed in the interrupt service routine thus enabling to send out the response much faster than if the processing was being carried out in the main cycle of the program. In this case speed is of great importance because once a wireless sensor sends out data, for a very brief period of time it switches to the listening mode, and waits for acknowledgment from concentrator, therefore fast response grants considerable energy saving. It takes $50 \mu \mathrm{s}$ for the concentrator to send out the acknowledge using the interrupt service routine.

If during this time the wireless sensor does not receive a response from the concentrator, a repeat sending is initiated thus contaminating the ether of the radio network and causing energy waste.

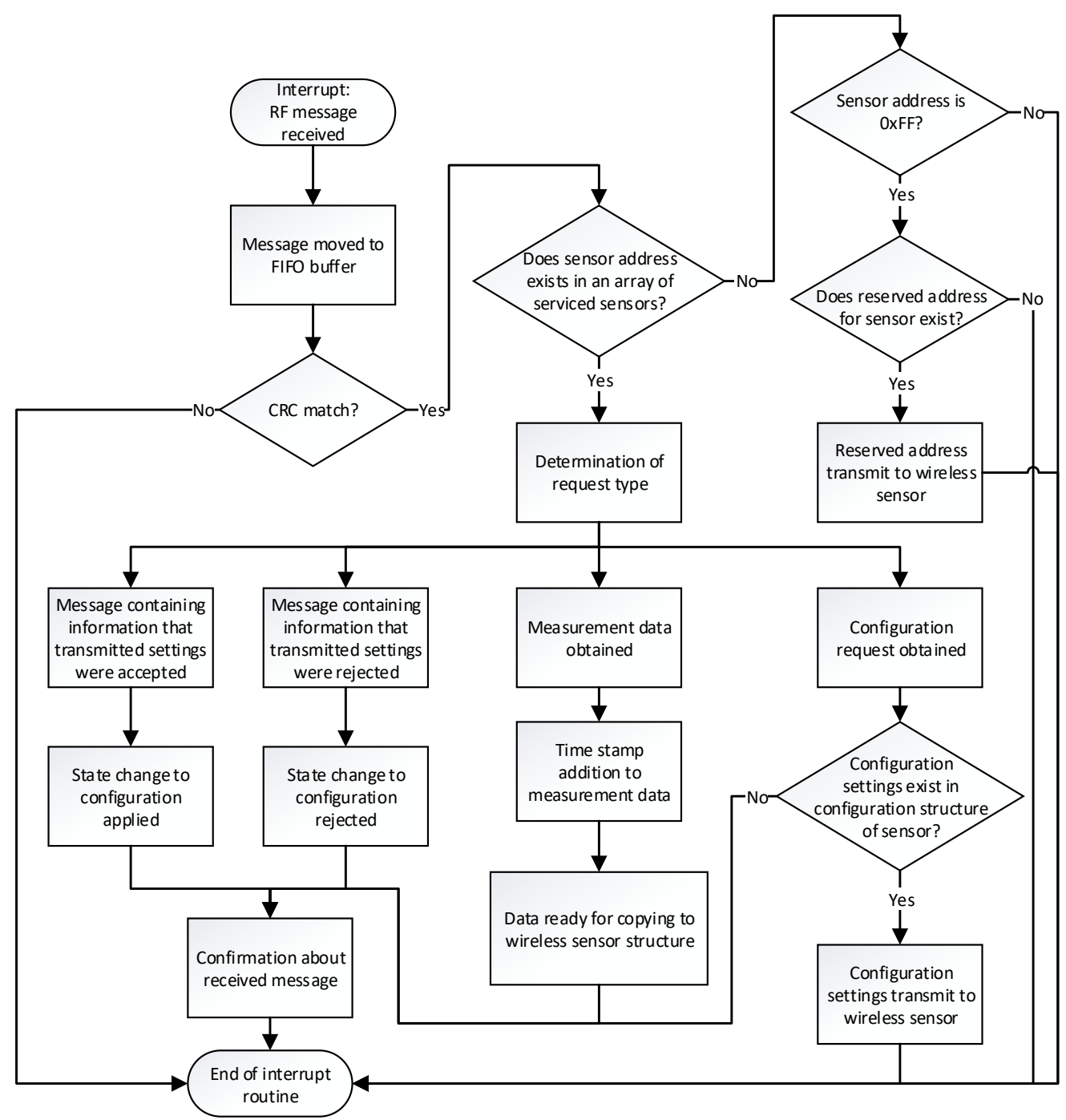

Figure 6. The algorithm of the interrupt initiated upon receiving a message via the radio network 
Each received measurement is processed in interrupt first. It is marked with a time stamp and an indication that the measurement is ready for further processing in the main cycle. Upon leaving the interrupt, the microcontroller program returns to the main cycle responsible for serving requests received via UART interfaces, processing measurements from the data structures, eliminating potential radio module disruptions and ensuring the functionality of the normal mode. Upon identifying that in the main cycle the data structures were supplemented with new results obtained from the measurements conducted in the wireless sensors, these are transferred to the relevant data storage structure by applying the necessary minor changes to the format and allocation. The concentrator automatically determines whether there are lost data packets. This process is carried out by calculating the time intervals between the received data. If these intervals exceed the period of time assigned to a wireless sensor, the meter of lost packages is increased. Upon receiving data from a wireless sensor this meter is being restarted. The second step involves the testing of the state of the A110LR09 module: it was observed that in case of a very high radio ether noise, there is a possibility to encounter the occurrence of radio module switching to the overload or other undesirable mode. To avoid any system failure, the radio module is regularly checked and, upon the identification of potential need, rebooted or otherwise brought back to the normal radio network listening mode.

\section{Experiments}

Upon creating the prototype of the system, various experiments were conducted. Their purpose was to establish the current used by a wireless sensor and the preliminary time of operation with the selected two $1200 \mathrm{mAh}$ capacity alkaline AA batteries. The current used by 
the wireless sensor in the sleep mode is provided in Figure 7. There are two main areas: one with running voltage booster and one with direct power feed.

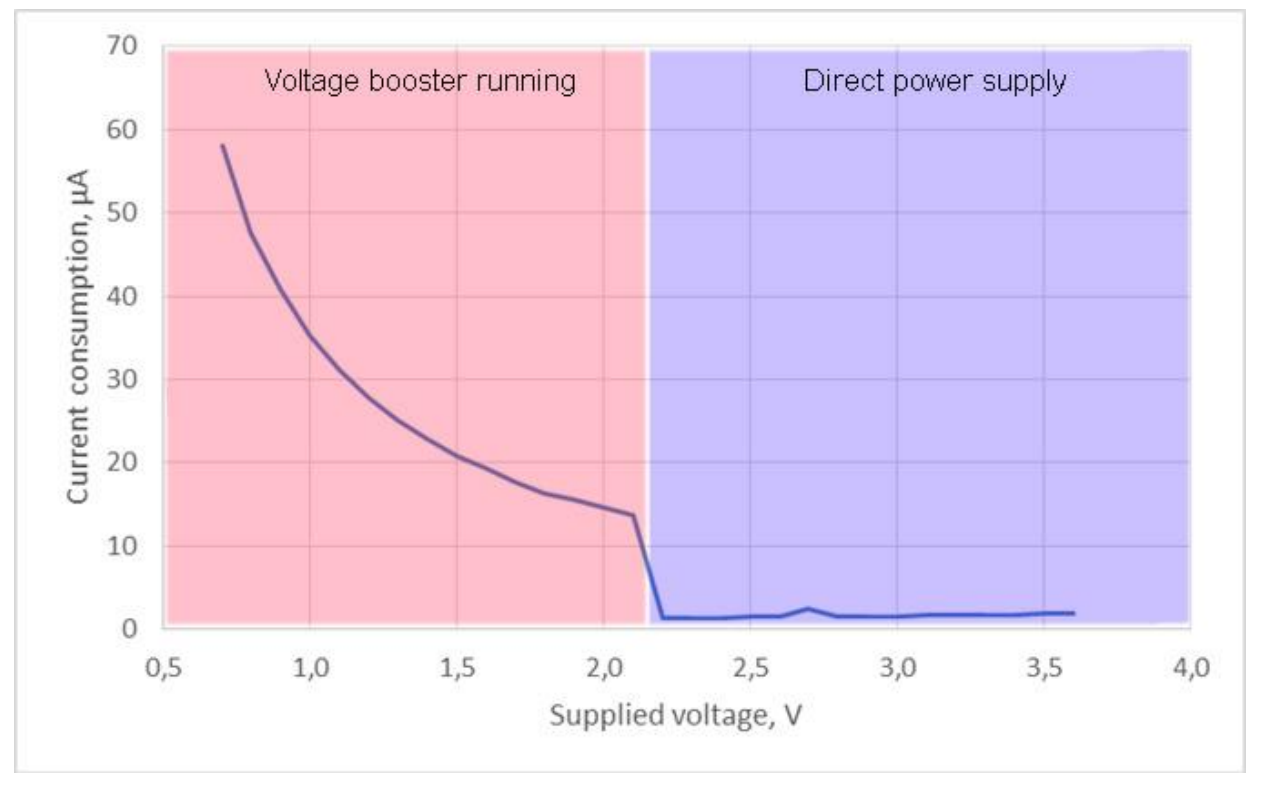

Figure 7. The dependence of the current used by a wireless sensor in the sleep mode on the supplied voltage.

The current starts to immediately increase once the voltage drops below $2.2 \mathrm{~V}$. This happens because a wireless sensor detects that the battery voltage is too low for the normal functioning, so it automatically switches the supply chain, i.e., the voltage multiplier is being turned on. It was observed the lowest required voltage had to be not smaller than $1 \mathrm{~V}$, further decrease of voltage cause sensor failure while starting up. With respect to the specifications of all elements used, the supply voltage cannot exceed 3.6 V. To sum up, the functionality of a wireless sensor is ensured when the power supply voltage is in-between $1 \mathrm{~V}$ and $3.6 \mathrm{~V}$.

The dynamic current consumption used when a wireless sensor is conducting measurements and sending data to the concentrator was measured by using a shunt resistor in serial to the power supply of whole sensor and by observing voltage drop on it. Temperature measurements were done using ADS1118 converter with a K type thermocouple. Compared to power used for transmitting data, conversion with ADS1118 is not draining considerable 
amount of energy ( $125 \mathrm{pWh}$ or $0.4 \%$ of whole consumed energy for one measurement). Measurements were conducted in two cases - in the presence of a sufficient signal level and when the concentrator was not accessible. Up to $2.5 \mathrm{~V}$ a $10 \Omega$ shunt resistor was used, whereas in all other cases - a $1 \Omega$ shunt resistor was selected. Such a decision was made because upon exceeding $2.5 \mathrm{~V}$, the current used tended to drop immensely and by increasing the shunt resistance it was possible to maintain a sufficient drop in voltage to ensure the necessary accuracy of integration. The graph obtained under sufficient signal level with the concentrator and with the $2 \mathrm{~V}$ battery voltage is provided in Figure 8 . Graph under same signal level with the $3 \mathrm{~V}$ battery voltage is provided in Figure 9 . The voltage patterns differ due to influence of pulse mode of the booster (Figure 8) and due to filtering capacity in the measuring channel in Figure 9.

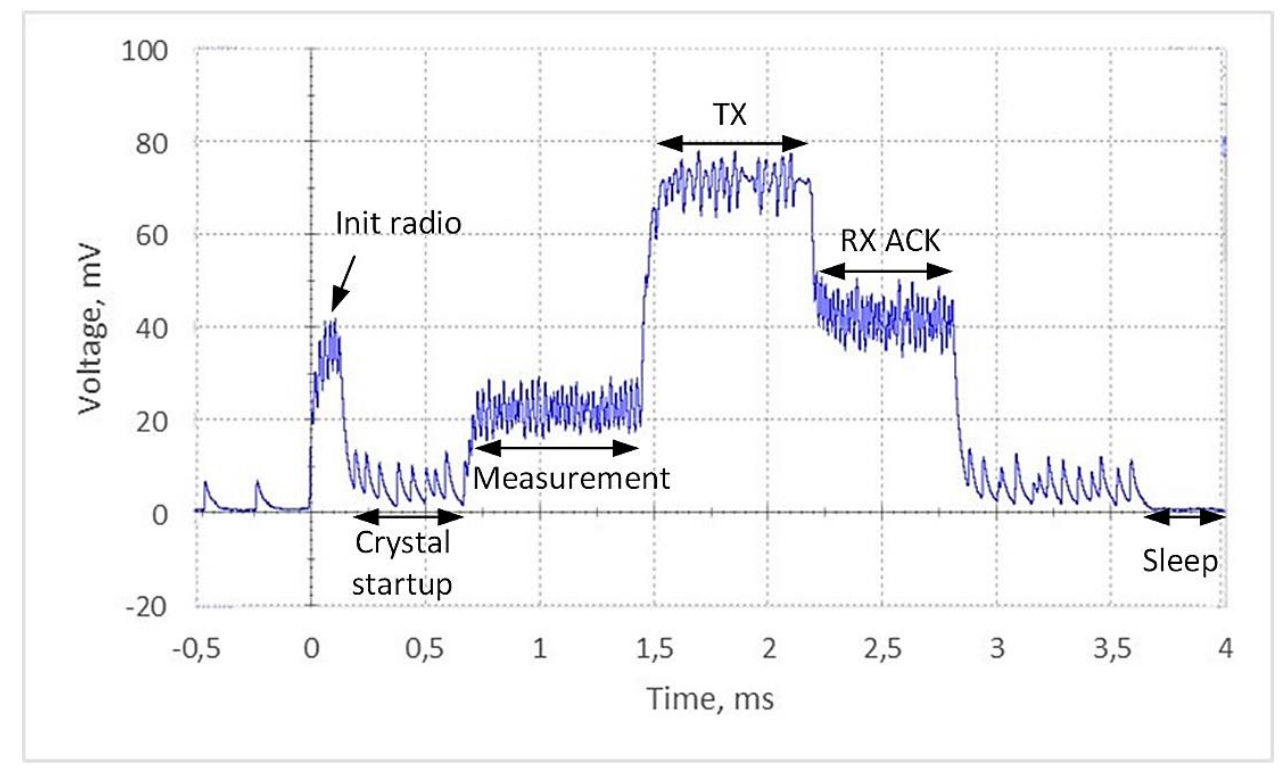

Figure 8. A drop voltage on the $1 \Omega$ shunt resistor when conducting temperature measurement and sending out data subject to the working voltage multiplier (signal level is sufficient, the concentrator is on, battery voltage is $2 \mathrm{~V}$ ). 


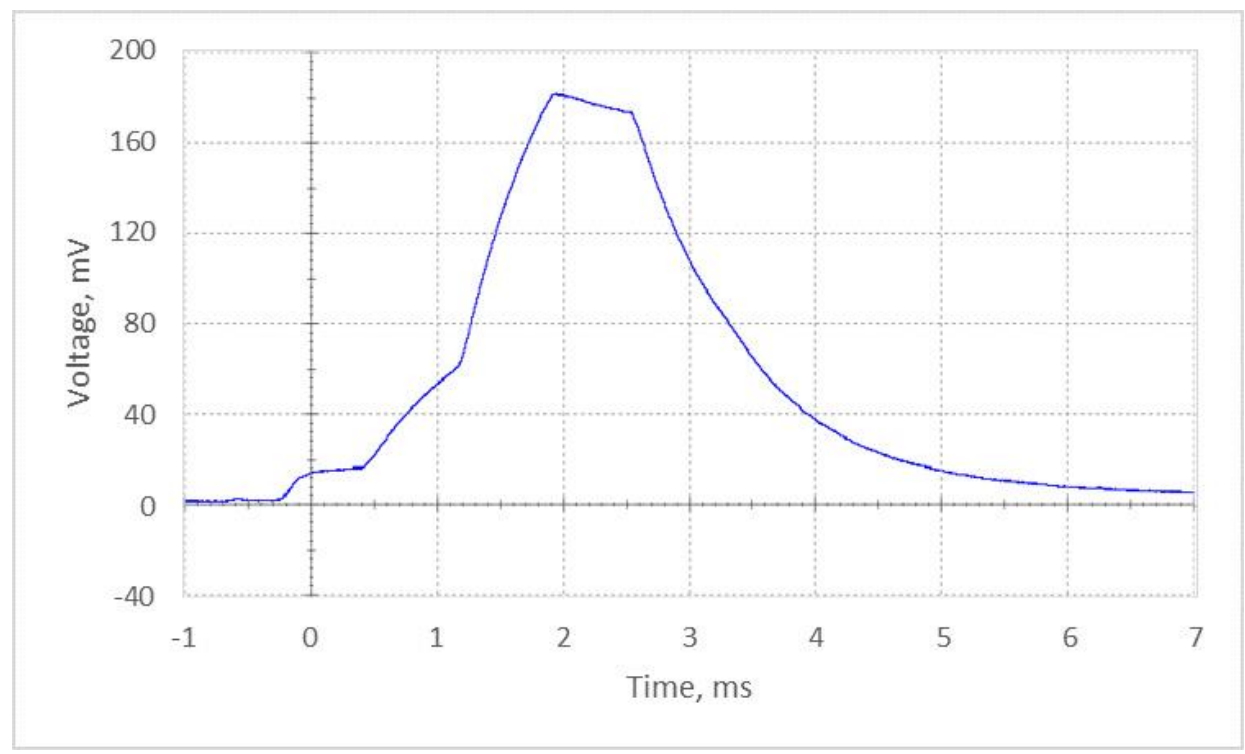

Figure 9. A drop voltage on the $10 \Omega$ shunt resistor when conducting temperature measurement and sending out data subject to the direct supply, the voltage multiplier is off (signal level is sufficient, the concentrator is on, battery voltage is $3 \mathrm{~V}$ ).

In case of absence of concentrator measurements are sent from wireless sensor predefined number of times until it is stated that there is no connection. At this point current consumption roughly multiplies by number of retries used. In order to determine energy consumption of one communication session voltage curve values (Figure 8 and Figure 9) were integrated. These calculations were done in various supplied voltage levels. Energy consumption dependence over supplied voltage is shown in Figure 10. There are two main areas: one with running voltage booster and one with direct power feed. Voltage booster turn on point is noticeably different than shown in Figure 7 due to extra voltage drop on shunt resistor. 


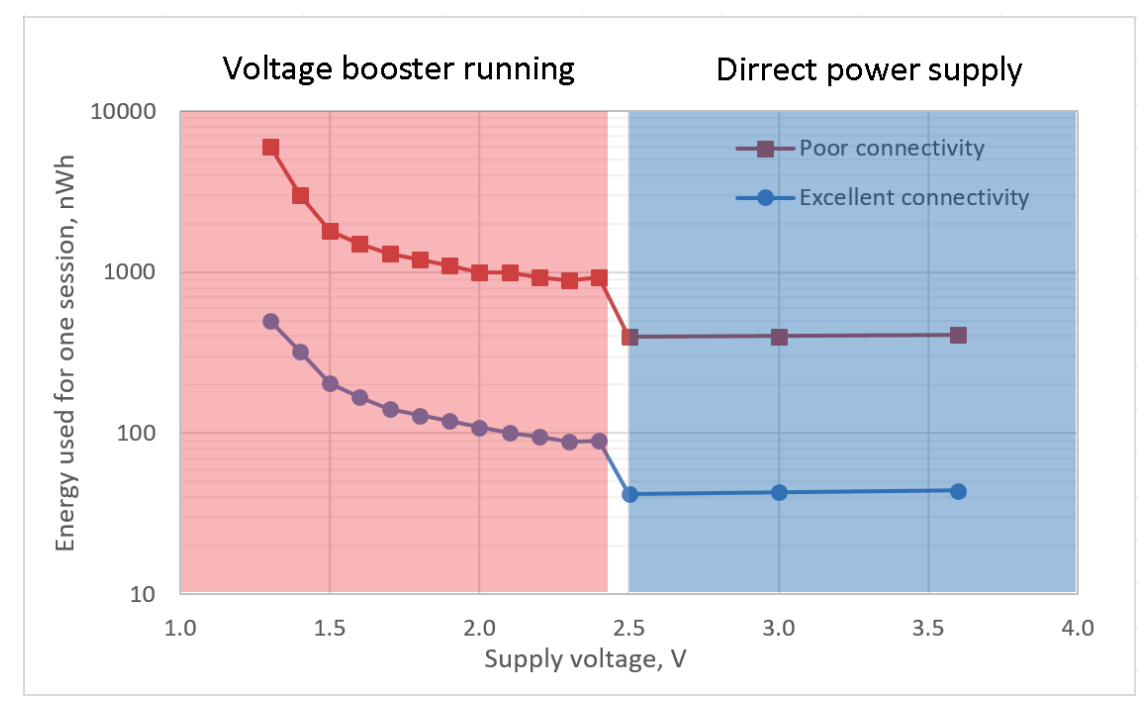

Figure 10. Energy usage for one communication session dependence over supplied voltage (in the case of poor connectivity the energy consumption is 10 times higher due to 10 connection retries).

Upon evaluating the obtained results, it has been determined that when conducting measurements every second a wireless network fed from two 1200 mAh capacity alkaline AA battery can operate for up to three years subject to sufficient signal level and up to seven months with no access to the concentrator. This difference is due to number of communication retries in case of poor connectivity (in our experiment number of retries was five ten). An increase in the period of measuring results in a prolonged period of operation. Upon conducting research into the maximum distance of operation the distance of 500 meters between a wireless sensor and the concentrator subject to direct visibility was determined.

The A 110LR09 module was programmed for a maximum radiated power of $10 \mathrm{dBm}$. The time of usage of this module for data sending was very short, i.e. $3 \mathrm{~ms}$. 


\section{Conclusions}

The system for gathering information from wireless sensor comprises a specific protocol and functionality algorithm which ensure the minimum operation time of the radio module. The prototype of the concentrator and the relevant highly effective microcontroller software which enables fast processing of the received data have been designed. Three independent communication interfaces - USB, RS-232 and RS-485 - operating in parallel mode and aimed at connecting different types of equipment have been foreseen. The support for Modbus RTU protocol enabling to read data using a computer and SCADA software or other compatible industrial equipment has been realized. The possibility to remotely monitor the state of wireless sensor feed elements, the quality of network and the loss of data has been realized. The methods of reducing and optimizing wireless network energy consumption have been thoroughly researched. The measured distance of wireless sensor operation subject to direct visibility is 150 meters. The experiment was conducted with 15 wireless sensors for 1 year.

Upon evaluating the obtained results, it is safe to say that when periodically conducting measurements every second a wireless network receiving energy from two $1200 \mathrm{mAh}$ capacity alkaline NiMH AA battery can operate up to three years and minimum one year when connectivity is excellent. Reducing temperature measurement frequency results in a proportional increase in operating time from limited power source.

\section{Acknowledgment}

This work was funded by a grant (No. SEN-10/15) from the Research Council of Lithuania. Project acronym: "CaSpine". 


\section{References}

1. Wireless Devices Deliver Great Benefits for Temperature Monitoring. Online: http://www.omega.com/techref/pdf/Wireless_Temperature.pdf

2. Ostaševičius, V.; Markevičius, V.; Jūrènas, V.; Žilys, M.; Čepènas, M.; Kižauskienė, L.; Gylienè, V. Cutting tool vibration energy harvesting for wireless sensors applications. Sensors and actuators A: Physical. 2015, vol. 233, pp. 310-318, doi: 10.1016/j.sna.2015.07.014.

3. Kaswan, A.; Nitesh, K.; Jana, P. K., Energy Efficient Path Selection for Mobile Sink and Data Gathering in Wireless Sensor Networks. AEU - International Journal of Electronics and Communications. 2017. doi: 10.1016/j.aeue.2016.12.005. (In Press).

4. Khan, P.; Ghosh, A.; Konar, G.; Chakraborty, N. Temperature and Humidity Monitoring through Wireless Sensor Network using Shortest Path Algorithm. In Proceedings of the International Conference on Control, Instrumentation, Energy and Communication (CIEC'2014). 2014, pp. 199-203, doi: 10.1109/CIEC.2014.6959078.

5. Jadidoleslamya, H.; Mohammad, R. A.; Bahramgiri, H, A Fuzzy Fully Distributed Trust Management System in Wireless Sensor Networks. AEU - International Journal of Electronics and Communications. 2016, vol. 70 iss. 1, pp. 40-49. doi: 10.1016/j.aeue.2015.09.017.

6. Markevicius, V.; Navikas, D.; Zilys, M.; Andriukaitis, D.; Valinevicius, A.; Cepenas, M. Dynamic Vehicle Detection via the Use of Magnetic Field Sensors. Sensors 2016, 16, 78. doi: 10.3390/s16010078. 
7. Safaria, L.; Minaeib, S.; Metin, B., A Low Power Current Controllable Single-Input Three-Output Current-Mode Filter Using MOS Transistors Only. AEU International Journal of Electronics and Communications. 2014, vol. 68 iss. 12, pp. 1205-1213. doi: 10.1016/j.aeue.2014.06.011.

8. Safaria, L.; Psychalinos, C., Two-Quadrant Fully Integrable RMS-to-DC Converter for Handling Low-Frequency Signals. AEU - International Journal of Electronics and Communications. 2015, vol. 69 iss. 12, pp. 1897-1901. doi: j.aeue.2015.09.002.

9. Daubaras, A.; Markevicius, V.; Navikas, D.; Cepenas, M.; Zilys, M.; Andriukaitis, D. Vehicle Influence on the Earth's Magnetic Field Changes. Elektron. Elektrotech. 2014, 4, 43-48. doi: 10.5755/j01.eee.20.4.4552.

10. Markevičius, V.; Andrijauskas, A.; Navikas, D.; Svensen, C.; Porvaneckas, N.; Andriukaitis, D.; Kvederas, G.; Cincikas, D.; Andrijauskas, P. Statistically biased calibration method for the real-time adjustment of noninvasive haemoglobin measurements in a semiautomated infusion system. Elektron. Elektrotech. 2013, 7, 65-71. doi: 10.5755/j01.eee.19.7.5165.

11. Joh, H.; Ryoo, I. A hybrid Wi-Fi P2P with bluetooth low energy for optimizing smart device's communication property. Peer-to-Peer Networking and Applications. 2015, vol. 8, iss. 4, pp. 567-577.

12. Zhu, L. Improved Probability Algorithm Based on Low Energy Bluetooth Technology. In Proceedings of the International Conference on Computer Science and Electronic Technology (ICCSET'2015), ShenZhen, P. R. China, 27-28 December 2014, pp. 155-157. 
13. Rault, T.; Bouabdallah, A.; Challal, Y. Energy efficiency in wireless sensor networks: A top-down survey. Computer Networks. 2014, 67, 104-122.

14. Touati, F.; Erdene-Ochir, O.; Mehmood, W.; et al. An Experimental Performance Evaluation and Compatibility Study of the Bluetooth Low Energy Based Platform for ECG Monitoring in WBANs. International Journal of Distributed Sensor Networks. 2015, Article ID 645781.

15. Dementyev, A.; Hodges, S.; Taylor, S.; Smith, J. Power Consumption Analysis of Bluetooth Low Energy, ZigBee and ANT Sensor Nodes in a Cyclic Sleep Scenario, 2013.

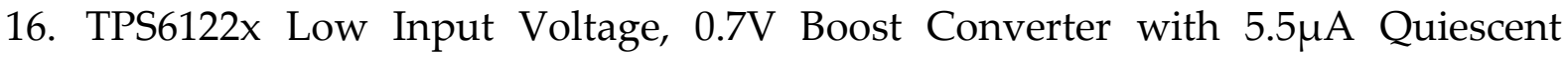
Current (Rev. B). Online: http://www.ti.com/lit/ds/symlink/tps61221.pdf

17. MSP430G2x53, MSP430G2x13 Mixed Signal Microcontroller (Rev. J). Online: http://www.ti.com/lit/ds/symlink/msp430g2553.pdf

18. Sinha R. S.; Wei Y.; Hwang S.H. A survey on LPWA technology: LoRa and NBIoT. ICT Express. 2017, 3, 14-21.

Vytautas Markevicius graduated MSc in 1973 and received PhD in Electronics Engineering in 1983. He works at Department of Electronics Engineering, Faculty of Electrical and Electronics Engineering, Kaunas University of Technology. The leader of the research group on Interactive electronic systems. His research focuses on finding solutions for the issues related to the interactive electronic systems, integrated information systems, energy harvesting, low power management, WSN.

Dangirutis Navikas graduated MSc in 1994 and received PhD in Electronics Engineering in 1999. He works at Department of Electronics Engineering, Faculty of Electrical and Electronics Engineering, Kaunas University of Technology. Also, he is a head of the Department of Electronics Engineering. His research focuses on finding solutions for the issues related to the interactive design of microprocessor systems, integrated information systems or WSN.

Darius Andriukaitis graduated MSc in2005 and received PhD in Electronics Engineering in 2009. He works at Department of Electronics Engineering, Faculty of Electrical and Electronics Engineering, Kaunas University of Technology. Also, he is a vice dean for research of the Faculty of Electrical and Electronics Engineering. His research focuses on finding solutions for the issues related to the interactive electronic systems, integrated information systems or WSN. 
Mindaugas Cepenas graduated from Kaunas University of Technology in 2012 with Master's degree in Electrical engineering. Currently, PhD study in Electrical and Electronics Department of Electronics Engineering studies KTU. Research areas - interactive design of microprocessor systems and low-power wireless networks.

Algimantas Valinevicius graduated MSc in 1979 and received $\mathrm{PhD}$ in Electronics Engineering in 1986. He works at Department of Electronics Engineering, Faculty of Electrical and Electronics Engineering, Kaunas University of Technology. Also, he is a dean of the Faculty of Electrical and Electronics Engineering. His research focuses on finding solutions for the issues related to the interactive electronic systems, integrated information systems or WSN.

Mindaugas Zilys graduated MSc in 1996 and received PhD in Electronics Engineering in 2001. He works as a researcher at Department of Electronics Engineering, Faculty of Electrical and Electronics Engineering, Kaunas University of Technology and in industry. His research focuses on electronic system efficiency, energy harvesting, low power management and wireless smart sensors.

Reza Malekian (PhD. CEng. FBCS), is an Associate Professor and Head of Advanced Sensor Networks Research Group at the Department of Electrical, Electronic and Computer Engineering, University of Pretoria. Dr. Malekian's research focuses on the Internet of Things (IoT), sensors technologies and smart cities.

Arturas Janeliauskas graduated MSc in1984 and received PhD in Electronics Engineering in 1995. He works at Department of Electronics Engineering, Faculty of Electrical and Electronics Engineering, Kaunas University of Technology. His research focuses on finding solutions for the issues related to the interactive electronic systems, smart environment and information technology.

Wojciech Walendziuk received his MSc degree in Electrical Engineering - Automation and Metrology in 1998, and PhD degree in Electrical Engineering in 2007, all from the Bialystok University of Technology, Bialystok. He is currently an assistant professor of Bialystok University of Technology, Faculty of Electrical Engineering, Department of Teoretical Electrotechnics and Metrology. He is a co-inventor of 2 national patents and the author of over 90 publications in the field of parallel processing, numerical methods, metrology, measurement of non-electrical quantities with the use of electrical methods. His main research interests include also signal analysis, signal conditioners and measurement systems application in medicine and Unmanned Aerial Vehicles.

Adam Idzkowski graduated at Bialystok University of Technology. He received MSc in Electronics and Telecommunications 2001, PhD in Electrical Engineering 2013. Currently, he works as an assistant professor at Bialystok University of Technology. He is the author or co-author of over 60 papers in the field of metrology, measurement systems and biomedical engineering, and a co-inventor of 2 national patents. 